\title{
Forest ecosystem genomics and adaptation: EVOLTREE conference report
}

\author{
Antoine Kremer • Barbara Vinceti • Ricardo Alia • Jaroslav Burczyk • \\ Stephen Cavers • Bernd Degen • Reiner Finkeldey • Silvia Fluch • Dusan Gömöry • \\ Felix Gugerli • Hans Peter Koelewijn • Jarkko Koskela • François Lefèvre • \\ Michele Morgante • Gerhard Mueller-Starck • Christophe Plomion • Gail Taylor • \\ Jozef Turok • Outi Savolainen • Birgit Ziegenhagen
}

Received: 16 December 2010 /Revised: 22 February 2011 /Accepted: 25 February 2011 /Published online: 24 March 2011

(C) The Author(s) 2011. This article is published with open access at Springerlink.com

\begin{abstract}
This article is a summary report of the international conference "Forest ecosystem genomics and adaptation" organized by the EVOLTREE Network of Excellence in San Lorenzo de El Escorial (Madrid), Spain, from 9 to 11 June 2010. Main achievements and results of the network are presented for the eight thematic sessions and a stakeholder session. The conference has shown that adaptive responses of trees to biotic or abiotic selection pressures can now be investigated at the gene level for traits of adaptive significance. Candidate genes have been catalogued for phenological and drought-related traits in important tree families (Salicaceae, Fagaceaea and Pinaceae), and their variation in natural populations is being explored. Genomics can now be integrated in ecological research to investigate evolutionary response to climate changes in a wide range of species. New
\end{abstract}

Communicated by S. González-Martínez

A. Kremer $(\triangle) \cdot$ C. Plomion

INRA, UMR Biogeco,

Cestas, France

e-mail: kremer@pierroton.inra.fr

B. Vinceti

Bioversity International, Forest Genetic Resources,

Rome, Italy

R. Alia

INIA, Forest Research Centre,

Madrid, Spain

J. Burczyk

KWUB, University of Bydgoszcz,

Bydgoszcz, Kuyavian-Pomeranian, Poland

S. Cavers

Centre for Ecology and Hydrology,

Edinburgh, UK avenues of research were also highlighted as the exploration of gene networks involved in adaptive responses and the combination of experimental and modelling approaches to disentangle components of evolutionary changes triggered by climate change. The main focus of the conference was the adaptation of trees to environmental changes. The conference was organized in eight thematic sessions ranging from genomic approaches aiming at identifying genes of adaptive significance to practical issues regarding mitigation options for combating climate change. A dialogue between scientists and end users took place in the form of an ad hoc stakeholder session. A panel of end users from various forest and policymaking institutions expressed their expectations, and the discussions with the scientists addressed the potential applications of research findings to the management of genetic

B. Degen

Bundesforschungsanstalt für Forst und Holzwirtschaft,

Grosshansdorf, Germany

R. Finkeldey

Forest Genetics and Forest Tree Breeding,

Goettingen University,

Göttingen, Lower Saxony, Germany

S. Fluch

ARC Seibersdorf Research GmbH,

Seibersdorf, Austria

D. Gömöry

Forestry, Technical University in Zvolen,

Zvolen, Slovakia

F. Gugerli

Swiss Federal Research Institute WSL,

Birmensdorf, Switzerland 
resources in the context of climate changes. The conference was introduced by two keynote speakers Dr. Pierre Mathy from the European Commission, Directorate General of Research, and Dr. Allen Solomon, former National Program Leader for Global Change, US Forest Service. All the thematic sessions were introduced by high-level invited speakers from the respective fields.

Keywords Adaptation - Genomics $\cdot$ Climate change

\section{Introduction}

The EVOLTREE Network of Excellence was launched in April 2006 as a European initiative to tackle the adaptive responses of trees and forest ecosystems to environmental change. The overall goal of the network is to link four major scientific disciplines (ecology, genetics, genomics and evolution) to address global issues that European forests are currently facing, such as environmental changes and erosion of biodiversity, and to instal the necessary resources, tools and infrastructures at the pan-European scale to support research in this field. On 9-12 June 2010, EVOLTREE organized an international conference, entitled "Forest ecosystem genomics and adaptation", during which the achievements of the network were presented in a broader context. The conference took place in San Lorenzo de El Escorial (Madrid), Spain, and it was attended by 230 participants from 37 countries. The event included eight thematic sessions ranging from genomic approaches aiming at identifying genes of adaptive significance to practical issues such as mitigation options for coping with climate change. A dialogue between scientists and end-users took place in the form of a special stakeholder session. A panel of stakeholders from various organizations responsible for forest management and policy making expressed their expectations, and the scientists explained the potential applications of their research findings to the management

\footnotetext{
${ }^{1}$ Abstracts of presentations by cited authors are available at this web site (index of authors at pages 237-242 of the book of abstracts).

$\overline{\text { H. P. Koelewijn }}$

Wageningen University and Research, WUR,

Wageningen, Netherlands

J. Koskela $\cdot$ J. Turok

International Plant Genetic Resources Institute,

Rome, Italy

F. Lefèvre

INRA, Unité de Recherches Forestières Méditerranéennes,

Avignon, France

M. Morgante

Universita degli Studi di Udine,

Udine, Italy

of forest genetic resources in the context of climate change. The conference was introduced by two keynote speakers (Dr. Pierre Mathy from the European Commission, Directorate General of Research, and Dr. Allen Solomon, former National Programme Leader for Global Change, US Forest Service); each thematic session was introduced by an invited speaker.

The book of abstracts and the presentations of the keynote and invited speakers are available at the EVOLTREE website (http://www.evoltree.org/index.php/evoltree-finalconference). ${ }^{1}$ Brief reviews of each session are presented in this conference report.

\section{Session 1: phenomics under climate change}

Phenomics attempts to characterize phenotypes of organisms at all levels that can be assessed, from gene expression to proteomics, from the metabolome through to quantification of complex adaptive traits and quantitative trait loci. Such traits include phenology, senescence, growth, photosynthesis and developmental characteristics, assessed in many ways, from counting and using a ruler to remote sensing using flight data. Over the last 5 years, during the progression of EVOLTREE, phenomics of forest trees has expanded dramatically, alongside the development of tools including cDNA and oligo arrays for non-model and model species, respectively, metabolomic and proteomic databases and platforms and finally, the application of re-sequencing technologies to transcriptomic quantification. The plenary speaker for this session, Stefan Jansson, described some ground-breaking work to understand the timetable for autumnal senescence such that the key regulators of bud set and senescence are now being identified, such as Constans and Flowering Locus T. We now know that the signalling mechanisms may be common between these two processes. In the context of climate change, much research is devoted to identifying genes for drought tolerance. In
G. Mueller-Starck

Fachgebiet Forstgenetik, Technische Universität München, Freising, Germany

G. Taylor

University of Southampton,

Southampton, UK

O. Savolainen

Department of Biology, University of Oulu, Oulu, Finland

B. Ziegenhagen

Conservation Biology, Philipps-University of Marburg, Marburg, Germany 
both Populus and Quercus, variation is present when contrasting genotypes are subjected to drought stress. Adaptation to southern European climates is clear in Populus. Viger et al. showed that when Populus nigra selected from the relatively wet northern Italian climate is subjected to drought stress; then, more than 3,000 genes are altered in transcript abundance. In contrast, for a Spanish poplar population selected from an area with approximately half of the rainfall, only a few hundred genes changed transcript abundance. This major re-programming of the transcriptome provides a rich source of candidate genes for association studies. Further research in Quercus on drought revealed growth compensation following re-watering, and up-regulated genes underpinning this response include those of the cell wall. This work may provide a significant resource for future breeding and improvement in Quercus.

Two important contributions on the responses of Populus to rising atmospheric $\mathrm{CO}_{2}$ and ozone showed changes in the transcriptome that could have important consequences for interacting herbivores. Secondary metabolic pathways may be induced in autumn following exposure to rising atmospheric $\mathrm{CO}_{2}$ (Tallis et al.), and these metabolites could protect the plants from herbivore attack. In contrast, ozone may impair the induced jasmonic acid pathway that is required for plant defence in response to insect attack (Herr et al.).

\section{Session 2: population genomics of adaptive traits}

The population genomics sessions (talks and posters) were preceded by a plenary talk by Magnus Nordborg of the Gregor Mendel Institute of Vienna. He described recent findings on Arabidopsis thaliana association studies with a genome-wide assay of SNPs. Questions of interpretation and the role of confounding effects in this selfing species with a high level of population structure were addressed and discussed. This provided an excellent comparison with studies in outcrossing, highly recombining forest trees, with much less neutral background population structure.

The work on population genomics has been advancing on several fronts. Forest tree genomic resources are increasing, even in species other than Populus, for which a full genome sequence is already available. The papers by Garnier-Géré et al. and Abadie et al. utilized recently developed Single Nucleotide Polymorphism (SNP) resources to analyse the divergence of the genomes of the hybridizing Quercus petraea and Quercus robur. This work will have the potential to identify the areas of the oak genome responsible for the partial reproductive isolation.

Increasingly, studies carefully control for demographic factors (such as population expansion or retractions), using, e. g. approximate Bayesian computation methods which are intensive computational techniques allowing to make evolutionary inferences. The extensive geographical sampling allows for the examination of environmental correlates of SNP variation (while simultaneously correcting for demographic history). Grivet et al. found significant correlations of candidate genes for drought with temperature in Mediterranean pines. Holliday et al. were able to account for much of phenotypic variation in phenology by clinally varying SNP frequencies. Concurrent clinal variation in Pinus sylvestris SNPs and timing of budset were presented in the poster session. Alberto et al. found that few of the individual associations or selection signals were repeatable across clines in a study of SNP variation and associations along two replicated altitudinal and latitudinal gradients in $Q$. petraea. These results emphasize the importance of the biological replicates.

So far, the results show moderate effects at individual SNPs, and low proportions of total genetic variance are explained. The ability to account for the phenotypic variation may increase when the candidate genes can be judiciously chosen, such as from the photoperiodic pathway genes in Picea, or if a large share of the SNPs in the total functional genome can be included. Increasing sample sizes and including interaction effects between loci may improve the ability to account for the variation as predicted by theory.

\section{Session 3: evolutionary responses to environmental change}

Variation in adaptive traits and its genetic basis have been investigated for decades, but a picture of the mechanisms resulting in evolution of trees in response to changing environments starts to emerge only now. Reviews on North American conifers (Pseudotsuga menziesii, Pinus taeda and others; Krutovsky et al.) and European aspen (Populus tremula, Ingvarsson) provide insights into patterns of adaptive variation including traits and their genes. However, allelic variation at single SNPs of putatively adaptive genes rarely explains a substantial percentage of the phenotypic variation in traits. This general finding points towards the complexity of evolutionary responses, likely involving numerous interacting loci, to changing environments.

The role of epigenetics in adaptive responses of tree populations to climate change conditions is poorly known. Experiments in Picea abies (Skrøppa et al.) showed that the reproductive environment has strong and lasting effects on the adaptive traits of spruce seedlings. The molecular basis of these results and its persistence after several generation cycles are not yet fully understood. Research in this field is urgent since epigenetic effects might extend adaptation to changing climatic conditions. 
The analysis of evolutionary responses to environmental change is further complicated by the need to consider all evolutionary factors and not only selection. Experimental studies on seed and pollen dispersal in various oak stands (Quercus spp.) show common trends but also important differences (Gerber et al.). The joint analysis of several evolutionary factors such as selection and gene flow requires the use of complex models (Alleaume-Benharira et al.). Experimental studies on adaptation to extreme environments indicate complex responses due to selection and other factors in pines exposed to high radiation in the Chernobyl exclusion zone (Kuchma et al.) and in oaks and beech at their xeric limits (Mátyás et al.).

The need for careful analysis of adaptive trade-offs between different traits was highlighted by the example of growth and reproductive traits in Mediterranean pines (Climent et al.). The view of large standing genetic variation as a prerequisite to adapt to different environmental conditions is challenged by the extremely low diversity at SSR and SNP markers in Pinus pinea (Vendramin et al.), while significant variation was observed at adaptive traits in provenance trials. The explanation of such discrepancies between phenotypic and molecular variation continues to be one of the greatest challenges for research on evolutionary responses to environmental change and for the population genomics of trees.

\section{Session 4: community responses to environmental change}

We are still far from understanding single species responses to environmental changes and much less the response of entire communities. Case-specific studies, ranging from species interactions to community ecology, were illustrated by a variety of presentations within this session. Paradigms of disturbance ecology such as resistance and resilience were revisited in the presentation by Kipfer et al. on $P$. sylvestris in Alpine forests. Thereafter, ectomycorrhizal (ECM) communities were considered key players to understanding the regeneration of $P$. sylvestris after standreplacing forest fires. Resilience of ECM communities was evidenced after 20 years of regeneration.

Most of the other presentations were focused on looking for the presence of "extended phenotypes" sensu Whitham et al. (2006). Several tree species were considered in relation with their associated above- and below-ground communities, namely insect herbivores and ECM communities. The analyses covered the genome, genotype and family level of individuals and populations under diverse environmental settings.

Francis Martin impressively summarized full-genome and transcriptome data on mycorrhizae in his keynote, putting up a landmark in tree-ECM interaction research. From this wealth of genomic data and subsequent hypothesis-driven experimentation, it became obvious that small secretory peptides play a key role during symbiosis formation.

At the family level, first results of DeWoody et al. indicate that particular regions within the poplar genome are involved in triggering associations with certain guilds of insects, not only affecting damage types, but also the timing of such damage. Further pioneering experiments provide evidence about genotype diversity effects on both insects and ECM communities. Though only preliminary results were available, Castagneyrol and Jactel found lower herbivory in mixtures of $Q$. robur genotypes than in single-genotype cultures after one growing season. Furthermore, indications for a host genotypic effect on the diversity and composition of ECM communities were presented by Karlinski et al. from common garden experiments with poplar clones. Translocation experiments with Q. robur genotypes (Cavers et al.) and studies on the wellknown poplar clone P. nigra var. italica (Tuba et al., Karlinski et al.) throughout several European countries attracted interest by pointing at the importance to environmental factors in tree-insect and tree-ECM interactions.

While the session topic was challenging, the approaches presented added empirical evidence of existing relationships between host genotype and associated communities, supporting current concepts of community genetics.

\section{Session 5: migration under climate change}

Rapid climate change puts species at risk of extinction by shifting the "climate envelope", beyond their current geographic range. The rate at which trees can extend their current ranges determines the magnitude of this risk. This session focused on recent findings from different approaches, such as simulation and statistical models, studies on dispersal and pollination ecology, which allow predictions about the way a changing climate will affect the migration patterns of trees.

Mapped networks of fossil pollen data for P. abies and Fagus sylvatica were compared with output from a climatebased vegetation model and a dispersal simulator (Bradshaw et al.). Both species must have had early outlying populations. Whilst $P$. abies responded directly to suitable climatic conditions, $F$. sylvatica also responded positively, but its north-eastern spread into Scandinavia was disjunct and frequently mediated by disturbance.

A landscape genetics study on $Q$. robur showed different patterns of pollen flow between oak populations in central Europe and eastern Russia (Buschbom et al.). Their results suggest that long-distance pollen transport can be very 
effective at the edge of the distribution. Small populations scattered across the landscape act as pollen traps. Quantifying long-distance pollen dispersal is a serious problem in studies of gene flow over large scales. To account for this difficulty, Burczyk et al. developed a new approach for estimating long-distance pollen immigration in $Q$. petraea and $Q$. robur stands, taking into account the change in flowering phenology between offspring and adults.

Using spatially explicit parentage models, Chybicki et al. investigated patterns of contemporary gene flow in different beech stands in central Europe. Pollen dispersal was similar for most of the stands while the scattered population in the Netherlands exhibited the highest pollen immigration rate of more than $70 \%$. In contrast, while seed dispersal was fairly restricted in natural and managed stands, it appeared to be high in one mountainous forest with more 30\% seed immigration.

Moran et al. used a stand simulator to estimate the effective seed dispersal for Quercus rubra derived from a hierarchical Bayesian parentage analysis incorporating genetic and ecological data. They found that competition with other forest species slows potential migration rate significantly relative to simulations without inter-specific competition.

From genetic data, it seemed that whilst tree species might be connected relatively efficiently over long distances via pollen dispersal, natural seed dispersal is probably too restricted to allow sufficiently rapid migration in the face of climate change and may cause tree populations to decline unless suitable management strategies are identified.

\section{Session 6: eco-regional trends in adaptation}

This session aimed at presenting ecological and phenotypic approaches to the study of adaptation under specific ecological settings. Graham Bell gave a keynote on the basic processes and requirements involved with adaptation to different environments. His message was that experiments are needed to study the processes of adaptation, and these are easier to establish with "simple" organisms. He started his talk with the challenging statement: "few, if any, of these requirements can be achieved with trees, because they are too big and too slow".

However, it was obvious from the session that conclusive experimentation can also be carried out with trees. During the past decades, foresters have established provenance trials all over Europe. These trials could be used for calculating the reaction norms of different populations and provide information on local adaptation. Four of the six submitted talks in the session dealt with data from provenance trials and reciprocal transplants. Thus, something can be done with trees, though not to the extent wished for by Graham Bell.

Stephen Cavers started off with considering the current distribution of $P$. sylvestris in Scotland from an environ- mental, genetic and historical perspective. The data indicated multiple origins for $P$. sylvestris in Scotland but showed clear patterns of phenotypic variation among populations. Bruno Fady discussed whether the hotspots of species diversity in the Mediterranean coincided with population genetic diversity in the same regions. He described a marked decrease in genetic diversity going from the east to the west of the Mediterranean, a pattern not congruent with that of species diversity.

Sylvain Delzon reported marked differences in the ecophysiological response of $F$. sylvatica and Q. petraea to an altitudinal gradient. Flushing time in Quercus responded strongly to a change in temperature, while Fagus hardly showed any difference. Thus, these two widespread broadleaves are expected to respond differently to climate change. Luis Santos-del-Blanco showed a clear trade-off between growth and reproduction in Pinus halepensis. Both talks were nice examples of what can be achieved when trees are moved around in their native range.

Finally, two more genomic-oriented talks concluded the session. Sally Aitken described the search for SNPs in candidate genes for drought and cold tolerance in Picea sitchensis. Felix Gugerli addressed the promises and pitfalls concerning the search for associations between ecologically important traits and genomic data. He highlighted the difficulties in finding the "adaptive islands" in the "sea of genomic neutrality" given spatial structure and demographic history.

\section{Session 7: new technologies in ecosystem genomics}

Advances in ecosystem genomic studies increasingly rely on progress in technology that enables to store and process an overwhelming amount of data. Second and third generation sequencing technologies are transforming ecosystem genomics, which now require advanced tools for data storage, analysis and data sharing via large-scale integration of distributed data sources, as demonstrated by D. Kopecky et al. Although NGS technologies provide the most advantages when reference genome sequences are available, they can greatly help to produce de novo assemblies as well as support transcriptome analysis and other assembly-free approaches.

Using Vitis vinifera as a model, Michele Morgante showed that the genome of two varieties can vary up to $25 \%$, due to small and large structural variations. To fully describe the genome of a species, it will be necessary to sequence multiple individuals from one species to generate the basis for a comprehensive catalogue of variation.

When working with species without a fully assembled reference genome, cross species and cross dataset comparisons allow novel insights in conserved regions 
of the genomes or transcriptomes of interest. Barakat et al. exploited datasets of closely related species of the Fagaceae to gain novel insights in evolutionary relationships among coding sequences. By combining Sanger and NGS data sets, comprehensive catalogues of transcribed genes are being developed for Picea as well as Quercus (S. Uneo et al.). When analysing conserved orthologous genes in Fagaceae and beyond, Yao-Cheng Lin et al. demonstrated structural changes (gains and losses of genes) throughout genome evolution within this plant family and pinpointed the evolution of new functions that occurred during speciation.

By applying these novel approaches, NGS might allow the sequencing of whole genomes, enabling to understand cause-effect relationships by combining sequence information with phenotypic data.

\section{Session 8: mitigation options}

This session was devoted to mitigation options available to cope with climate change. It was concluded that assisted migration as a mitigation strategy needs to be reconsidered due to species interactions between plants and plantdependent organisms such as insects.

The presentations and posters covered essentially two broader issues: (1) common garden experiments and their implications for the movement of forest reproductive material and (2) maintenance of genetic diversity as a prerequisite for retaining adaptability of forest tree populations. For the first topic, examples of provenance trials on several forest tree species (P. sylvestris, $Q$. petraea, Fagus sylvatica, Abies alba) highlighted potential consequences of climate change for the distribution and vitality of tree populations, depending on particular climate change scenarios. Several experiments demonstrated that the local sources do not necessarily perform best. The lack of superiority of local provenances indicates a rather limited extent of local adaptation in beech. At the same time, strong genotype-by-environment interactions appear to be a general phenomenon in forest trees, making it difficult to give general practical recommendations for measures regarding climate change mitigation.

The presentations showed that the current provenance regions cannot always be justified by the latest studies. Provenance experiments with large numbers of origins tested at sufficient numbers of sites can represent an efficient tool for defining adaptively homogeneous areas. Growth and adaptive traits such as timing of bud burst or growth cessation were proposed as a suitable basis for guidelines on the transfer of reproductive material.

Conservation of genetic resources, including ex situ practices, constitutes an indispensable component of measures aimed at mitigating the impacts of climate change. Within the group of presentations concerning the second topic, a novel approach was introduced to determine the effective number of mother trees used for seed collections to maintain a sufficient representativeness of ex situ collections. The method developed for $P$. sylvestris is potentially extendable to other windpollinated coniferous species. In respect to in situ conservation, silvicultural practices based on natural regeneration ensure the preserving of genetic variation present in forest stands although different silvicultural treatments may have different consequences for spatial genetic structures in beech stands.

Discussions pointed out that caution is necessary when recommendations for active mitigation measures are based solely on growth responses and juvenile survival assessed in provenance experiments, especially when uncertainty of the predictions of future climate development is considered. The role of phenotypic plasticity and epigenetic phenomena is frequently underestimated in the predictions of future distributions of tree species.

\section{Stakeholder session}

The stakeholder session started with two presentations. Bruno Fady demonstrated how forest ecosystem genomics research can contribute to conservation of tree genetic resources, and Bernd Degen presented a case study on how forest ecosystem genomics can support the appropriate use of forest reproductive material and certification of its origin.

The presentations were then followed by panel discussions. The panellists expressed a strong need for answers to the following questions: (1) how should existing forests be managed to maintain their adaptation capacity to climate change and (2) how should forest reproductive material be used in the face of climate change? They also pointed out that provenance regions should be re-designed and harmonized at the pan-European level. Furthermore, they noted that a pan-European scheme for certification of the origin of forest reproductive material would be very useful. The need for indicators to monitor the genetic diversity of forest trees was also raised.

Moreover, it was emphasized that the forest sector needs to pay more attention to access and benefit sharing (ABS) issues related to forest genetic resources. A new international agreement on ABS is currently being negotiated under the Convention on Biological Diversity. The implications of the final ABS agreement for the use of forest reproductive material across countries, tree breeding and collection, exchange and storage of genetic material for research purposes should be carefully evaluated. 
The stakeholders pointed out that coordinated international efforts are needed for conservation of forest genetic resources. National programmes or strategies on forest genetic resources are important tools and building blocks for which international collaborative efforts, such as the European Forest Genetic Resources Programme, can build on their activities. It was also recognized that the results of the EVOLTREE Network of Excellence, in particular the common research infrastructures for ecosystem genomics research, offer significant benefits for the future work on conservation and use of forest genetic resources.

The representative of the pan-European forest policy process (Forest Europe) welcomed the scientific community to contribute to thematic issues that will be addressed by the sixth ministerial conference, to be held in Oslo in June 2011. These issues include loss of biodiversity, adaptation of forests and forest management to climate change, and illegal logging. The science-policy dialogue is also an integral part of the Forest Europe process, and the scientific community has been involved in the process since its inception in 1990.

In their concluding remarks, the stakeholders expressed their gratitude to EVOLTREE for making serious efforts to engage them in the dialogue and hoped that the efforts will be continued.

\section{Concluding remarks}

The conference has clearly shown that adaptive responses of trees to biotic or abiotic factors can now be investigated at the gene level for traits of adaptive significance. Candidate genes have been catalogued for phenological and drought-related traits in important tree families (Salicaceae, Fagaceaea and Pinaceae), and their variation in natural populations is being explored. Genomics can now be integrated in ecological research to investigate the evolutionary response to climate changes in a wide range of species. However, the emerging results presented in population genomics and association studies suggest that adaptation is triggered by genome-wide changes and interactions among many genes. Tracking of adaptive genomic imprints could be foreseen with the development of second (third) generation sequencing methods allowing the exploration of genomewide population differentiation in an increasing number of samples. This exploration should also be prolonged beyond single locus approaches and target interactions and covariations between different candidate genes. Future research should therefore integrate these findings into new approaches, some of which were highlighted during the conference. It was suggested to go beyond the traditional retrospective dissection of population differentiation in common garden experiments, and to implement experimental research in controlled ecological settings. An important issue is whether rapid local adaptation builds on standing genetic variation or may also be fuelled by "immigrant" genes via long-distance gene flow. As discussed during the "migration" session, contemporary gene flow should therefore be assessed at landscape scales. It was also suggested to monitor evolutionary changes at the phenotypic and genomic levels in appropriate experiments (including contemporary population transfer from known source populations) allowing to separate tree responses due to natural selection and phenotypic plasticity. Modelling is another avenue to explore and an opportunity to integrate genetic, ecological and evolutionary mechanisms to understand and predict the responses of tree population to climate change.

Acknowledgements We are grateful to the different institutions and organizations involved in the conference preparation, for their technical and financial support: the European Commission (DG 12), Bioversity, INIA, INRA, INRA Transfert and the EVOLTREE Network of Excellence. We are also grateful to the members of the organizing and scientific committees for their contribution to the event.

Open Access This article is distributed under the terms of the Creative Commons Attribution Noncommercial License which permits any noncommercial use, distribution, and reproduction in any medium, provided the original author(s) and source are credited.

\section{Reference}

Whitham TG, Bailey JK, Schweitzer JA, Bangert RK et al (2006) A framework for community genetics: from genes to ecosystems. Nat Rev Genet 7:510-523 